\author{
Artem SMIRNOV ${ }^{1}$ \\ Lucia ANEMONA ${ }^{1}$ \\ Manuela MONTANARO ${ }^{1}$ \\ Alessandro MAURIELLO ${ }^{1}$ \\ Margherita ANNICCHIARICO- \\ PETRUZZELLI ${ }^{2}$ \\ Elena CAMPIONE ${ }^{3}$ \\ Gerry MELINO ${ }^{1,4}$ \\ Eleonora CANDI ${ }^{1,2}$ \\ ${ }^{1}$ Department of Experimental Medicine, \\ TOR, University of Rome "Tor Vergata", \\ 00133 Rome, Italy \\ 2 Istituto Dermopatico \\ dell'Immacolata-IRCCS, 00163 Rome, Italy \\ ${ }^{3}$ Department of Dermatology, University of \\ Rome "Tor Vergata", 00133 Rome, Italy \\ ${ }^{4}$ MRC-Toxicology Unit, University of \\ Cambridge, UK
}

Reprints: E. Candi

<candi@uniroma2.it>

Article accepted on 30/05/2019

\section{Transglutaminase 3 is expressed in basal cell carcinoma of the skin}

\begin{abstract}
Background: Transglutaminase 3 (TG3) belongs to a family of $\mathrm{Ca}^{2+}$ dependent enzymes which catalyse protein crosslinking. TG3 is important for proper development of the skin and hair shaft, and knockout mice for the Tgm3 gene are sensitive to UVB-induced photodamage due to aberrations in cornified envelope formation. Loss of TG3 is reported in head and neck and oesophageal squamous cell carcinoma, yet, its expression in skin cancer has not been studied. Objectives: The aim of the present study was to analyse the expression pattern of TG3 in skin cancer. Materials and methods: TG3 expression was investigated based on immunohistochemical staining of a tissue micro-array of different types of skin cancer, as well as meta-analysis of public gene array data. Results: Our findings demonstrated that TG3 is normally expressed in spinous/granular layers of the epidermis, but is absent in melanocytes as well as melanoma samples. As expected, its expression was absent in poorly differentiated squamous cell carcinoma of the skin. Surprisingly, we show that samples of basal cell carcinoma demonstrated strong staining for TG3 both in the cytoplasm and nucleus. Furthermore, at the mRNA level, the expression pattern of TGM3 was crucially altered in BCC, but not other types of skin cancer. Conclusion: These findings lead to new questions regarding TG3 involvement in basal cell carcinoma tumourigenesis. Moreover, the expression pattern of TG3 renders it a potential specific marker for basal cell carcinoma diagnosis.
\end{abstract}

Key words: basal cell carcinoma, keratinocytes, melanoma, skin cancer, squamous cell carcinoma, transglutaminase 3
$\mathrm{T}$ he family of transglutaminases (TGs) is composed of $\mathrm{Ca}^{2+}$-dependent enzymes whose main function is to catalyse protein crosslinking [1]. Transglutaminase 3 (TG3) is expressed in the differentiated layer of the epidermis and hair follicle. During keratinocyte differentiation, similar to the other TG members, TG1 and TG5, [2-4], proteolysis is required for TG3 activation [5]. It was demonstrated that Sp1 and ETS transcription factors promote TG3 gene (TGM3) expression in squamous epithelia [6], moreover, two different transcription variants of TGM3 were identified [7]. The main protein substrates for TG3 and TG1 are loricrin, involucrin, and the family of small proline-rich proteins (SPRs). Crosslinking between loricrin and SPRs contributes to the cornification of the skin [3]. Even though $\mathrm{Tgm}^{-{ }^{--}}$mice do not show any strong abnormalities in skin formation, a more invasive percutaneous penetration of fluorescein isothiocyanate (FITC) in KO mice was observed [8]. Recently, our laboratory demonstrated that the absence of $\mathrm{Tg} 3$ sensitizes the skin to damage induced by UVB irradiation [9]. Moreover, a mutation within the TGM3 gene was shown to be one of the causes of uncombable hair syndrome [10].

Multiple studies have demonstrated the downregulation of TG3 both at the RNA and protein level in head and neck squamous cell carcinomas [11-17]. As a molecular mecha- nism of TG3 repression, hypermethylation of $\mathrm{CpG}$ islands within the TGM3 promoter has been proposed [18]. Furthermore, TGM3 was shown as a putative tumour-suppressor gene in oesophageal cancer, in which its expression was downregulated and associated with tumour proliferation and migration $[19,20]$.

Skin cancer is the most common type of cancer. There are three different major types of skin cancer: basal cell carcinoma, squamous cell carcinoma (referred to as nonmelanoma skin cancer), and malignant melanoma. Basal cell carcinoma is the most common type of cancer in the world. It preferentially arises from stem cells of the hair follicle [21], meanwhile squamous cell carcinoma arises from epidermal keratinocytes and can be more aggressive and lead to metastasis [22]. Melanoma arises from melanocytes and its incidence is minor relative to non-melanoma skin cancers, however, it is the deadliest form of skin cancer [23]. Recently, a single nucleotide polymorphism was found within the TGM3 gene, associated with a higher risk of basal cell carcinoma incidence among Icelanders [24]. However, the expression of TG3 in skin cancers has not yet been investigated.

Here, using a tissue micro-array (TMA) approach combined with meta-analysis of skin cancer data sets, we investigated TG3 expression in different patients. We observed 
TG3 down-regulation in melanoma and aggressive squamous cell carcinomas, but a high level of expression in basal cell carcinoma, making TG3 a suitable biomarker for this type of skin cancer.

\section{Materials and methods}

\section{Immunohistochemical staining}

A skin cancer tissue micro-array, containing 10 samples of normal skin, 10 samples of malignant melanoma, 39 samples of cutaneous squamous cell carcinoma, and 13 samples of cutaneous basal cell carcinoma, was purchased from US Biomax (Cat. No. SK801c, Rockville, MD, USA). Other samples of cBCC from this study were utilized with the approval (Protocol No. 130/18) of the institutional review board of the University Hospital "Policlinico Tor Vergata" (Rome, Italy) and prior patient consent. The immunohistochemical staining for Ki67 and Ep-CAM were performed using anti-Ki67 antibody (Cat. No. 790-4286, Ventana, Oro Valley, AZ, USA) and anti-Ep-CAM antibody (Cat. No. 760-4383, Ventana), following the manufacturer's indications using the automatised BenchMark ULTRA slide staining system (Ventana). For TG3 staining, sections were dewaxed and rehydrated, incubated for blocking of endogenous peroxidases in $0.03 \%$ solution of hydrogen peroxide in methanol, and antigen retrieval was then performed by boiling the sample in the $0.01 \mathrm{M}$ citrate buffer $\mathrm{pH} 6.0$ for 10 minutes in a microwave. Slides were incubated with anti-TG3 antibody (1:300; Cat. No. C2D, Covalab, Villeurbanne, France) for 20 minutes at room temperature. The signal was detected using UltraTek HRP anti-polivalent DAB staining system (ScyTek, Logan, UT, USA) and the slides were then counterstained with haematoxylin, dehydrated, and mounted. Slides were scanned using a Ventana iCoreo scanner (Ventana) with 40x objective. Images of haematoxylin/eosin staining for TMA were downloaded from the manufacturer's website (www.biomax.us).

\section{Immunofluorescence and confocal microscopy}

Paraffin-embedded sections of BCC were dewaxed and rehydrated, and antigen retrieval was then performed by boiling the sample in $0.01 \mathrm{M}$ citrate buffer $\mathrm{pH} 6.0$ for 10 minutes in a microwave. To reduce tissue autofluorescence, samples were incubated for 45 minutes in $0.1 \mathrm{M}$ sodium tetrahydroborate solution, followed by one hour of blocking with 5\% goat serum in PBS. Slides were incubated with anti-TG3 antibody (1:300) and anti-Loricrin (1:300; Poly19051, BioLegend, San Diego, CA, USA) overnight at $4{ }^{\circ} \mathrm{C}$. Then, sections were incubated for one hour at room temperature with secondary anti-mouse and anti-rabbit 488- or 568-AlexaFluor conjugated antibodies (1:1,000; Invitrogen, Carlsbad, CA, USA) together with $1 \mu \mathrm{g} / \mathrm{mL}$ DAPI (Sigma, St. Louis, MO, USA) for nuclear DNA staining. Sections were analysed with a confocal laser microscope (NIKON Eclipse Ti) using NIS-Elements AR Ver. 4.4 software (Nikon, Tokyo, Japan).

\section{Histological scoring of the samples}

Samples were scored in a blinded manner by a pathologist using a semi-quantitative method. Cases were analysed for staining intensity, which was scored as 0 (not detected), $1+$ (weak), 2+ (intermediate), and 3+ (strong). For each case, the histological "H-score" (0-300) was calculated by multiplying the percentage of positive cells $(0 \%-100 \%)$ by the intensity (0-3). Percentage of Ki67 positive samples was calculated as the number of Ki67 positive neoplastic cells per total number of neoplastic cells $x 100 \%$. Ten random fields were analysed for each sample.

\section{Bioinformatic analysis}

Normalized values for TGM1, TGM3, TGM5, LOR, and IVL expression in the skin cancer samples were obtained from NCBI GEO portal (accession number: GSE7553 [25]). Analysis of co-expression was performed using the publicly available on-line platform "R2: Genomics Analysis and Visualization Platform" (r2.amc.nl). Gene ontology analysis was carried out using the DAVID on-line platform (david.ncifcrf.gov).

\section{Statistical analysis}

All statistical analyses were performed using GraphPad Prism 7.0 software (San Diego, CA, USA). For the analysis of gene array data and TG3 protein level from the tissue micro-array experiment, the significance level $(p)$ was calculated using Welch's unequal variances $t$-test. Values of $p<0.05$ were considered significant. Violin plots were generated in $\mathrm{R}$ using ggplot2 package.

\section{Results}

\section{TG3 is differentially expressed in skin cancer}

Previously published data revealed an important role of TG3 in oesophageal and head and neck cancer [14, 19], meanwhile its expression pattern in skin cancer remains unveiled. To analyse the expression of TG3 at the protein level, we used a tissue micro-array containing normal skin, cutaneous melanoma, squamous cell carcinoma (SCC), and basal cell carcinoma (BCC). Firstly, we performed immunohistochemical staining of TG3 using 10 samples of normal human skin (figure 1A). We observed a homogeneous distribution of the staining among all specimens regardless of the patients' age, sex, or biopsy anatomical site (supplementary table 1). In line with previous studies [26], all samples showed localisation of TG3 in the upper spinous and granular layers, meanwhile basal and cornified layers, as well as the dermis, were negative for TG3. As a marker of proliferation, we used Ki67. We then analysed the expression of TG3 in tumour samples; almost all samples of melanoma were negative for TG3 staining (figure 1B, supplementary table 2). Based on analysis of TG3 expression in the SCC samples (figure 1C, supplementary table 3), the majority of samples (24/39) were negative for TG3. Several samples of low-grade and well-differentiated tumours showed positive staining only in the differentiated cells relative to the poorly-differentiated samples. Surprisingly, we found a very heterogenous distribution of TG3 expression in the BCC samples (figure 1D, supplementary table 4); several samples were negative (4/13) or weakly-stained (4/13) for TG3, and almost half showed a very strong staining 


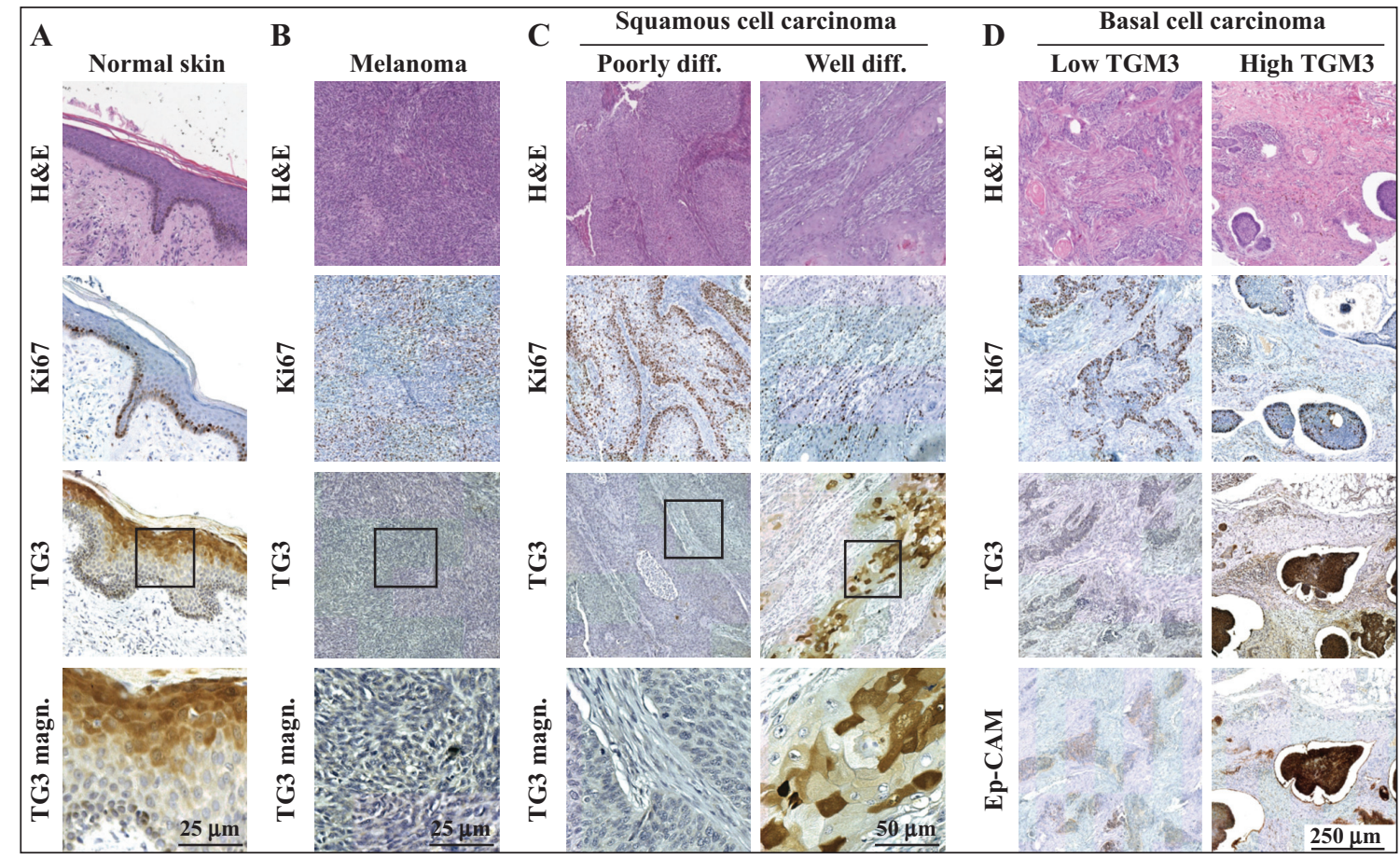

Figure 1. TG3 is differentially expressed in skin cancer based on tissue micro-array: H\&E staining and immunohistochemical analysis of Ki67 and TG3 expression in (A) normal skin $(n=10)$, (B) cutaneous melanoma samples $(n=10)$, and $(\mathbf{C})$ cutaneous squamous cell carcinoma $(n=39)$ (two representative cases of poorly- and well-differentiated tumours are shown). D) H\&E staining and immunohistochemical analysis of Ki67, TG3, and Ep-CAM expression in cutaneous basal cell carcinoma $(n=13)$ (two representative cases with low and high levels of TG3 expression are shown).

(5/13). However, more precise analysis of the samples in a blinded manner by three independent pathologists (University Hospital "Policlinico Tor Vergata", Rome, Italy) did not result in definitive conclusions regarding the diagnosis of TG3-negative samples. To resolve this problem, we performed immunohistochemical staining for Ep-CAM, which was shown to be a specific marker for basal cell carcinoma of the skin [27]. Surprisingly, only some samples $(4 / 13)$ were positive $(\mathrm{H}$-score $>100)$ for this protein (supplementary table 4). Hence, we decided to perform additional staining for six BCC sections from the Pathology unit of "Policlinico Tor Vergata" Hospital (Rome, Italy). We found all samples to be strongly positive for both EpCAM and TG3 (figure 2A). Interestingly, in TG3-positive samples, localisation was not only cytoplasmic but also nuclear. Further statistical analysis of TG3 expression based on the skin cancer tissue micro-array and additional cases (figure $2 B, C$ ) revealed a strong decrease in TG3 level in melanoma $\left(p=5.3 \times 10^{-6}\right)$ and $\operatorname{SCC}\left(p=5.9 \times 10^{-6}\right)$ samples relative to the normal epidermis. On the other hand, the TG3 level in the BCC samples (only Ep-CAMpositive samples from TMA and additional cases) was significantly increased relative to the normal epidermis $\left(\mathrm{FC}=2.2 ; p=6.5 \times 10^{-5}\right)$.

\section{Differentiation-related profile of TGM3 expression is lost in $\mathrm{BCC}$, but not in SCC}

For further confirmation of our observations, we analysed the expression of TGM3 at the mRNA level in normal skin and skin cancers from a publicly available gene array (accession number: GSE7553, [25] (figure 3A). We observed that TGM3 expression is significantly decreased in melanoma samples $(p=0.026)$, meanwhile there were no significant changes in TGM3 expression in SCC $(p=0.557)$. In line with our results, we observed a significant two-fold increase in TGM3 level in basal cell carcinoma samples $(p=0.011)$. Hence, we decided to investigate whether the expression of other skin-related TGs (such as TGMI and TGM5) or their common substrates (such as LOR and IVL) was altered in BCC relative to normal skin. Our results showed no significant changes in the expression of any of the genes analysed $(p>0.15)$, indicating that increased expression of TG3 in BCC is specific and not linked to a de-regulation of the differentiation process (figure $3 B$ ). To confirm these data at the tissue level, BCC samples were costained for TG3 and its substrate, loricrin. As highlighted in figure $3 C$, TG3 and loricrin were partly co-expressed in the upper layers of the skin adjacent to the tumour, meanwhile the tumour regions were positive for TG3 and completely negative for loricrin. To support the observation that TG3 is uniquely overexpressed in BCC, irrespective of other differentiation-related genes, we performed correlation analysis of expression of TGM1, TGM3,TGM5, LOR, and $I V L$ in normal skin and skin cancer using the data from the gene array (GSE7553). As expected, we observed a high correlation $(R>+0.75)$ between the expression of all genes analysed in normal skin, melanoma, and SCC samples (figure 4A). Surprisingly, in BCC samples, we found that this trend was maintained only between $T G M 1, T G M 5$, $L O R$, and $I V L(R>+0.75)$, but not TGM3 $(\mathrm{R} \approx 0)$. The gene 


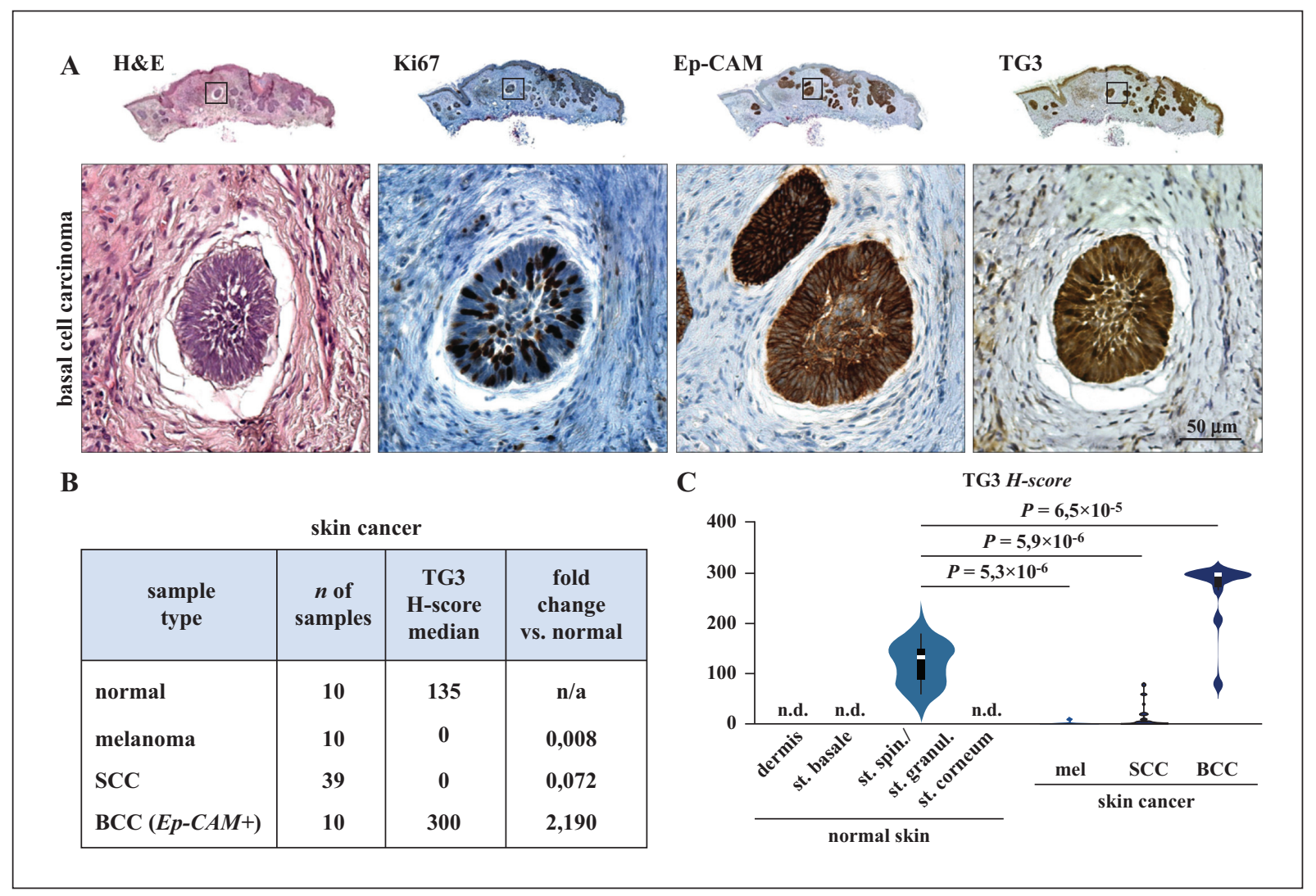

Figure 2. TG3 is highly expressed in Ep-CAM+ basal cell carcinoma. A) H\&E staining and immunohistochemical analysis of $\mathrm{Ki67}, \mathrm{TG} 3$, and Ep-CAM expression in cutaneous basal cell carcinoma $(n=6)$. B) Table showing the median H-score for TG3 and fold change in TG3 expression in tumour samples relative to normal skin. C) Violin plot showing TG3 H-score distribution in the samples of normal skin and skin cancer from $(\mathbf{B})$.

ontology analysis (figure $4 B$ ) revealed that the top 300 genes co-expressed with the TG substrate, LOR, are strongly related to an epidermal differentiation cluster in all types of skin cancer $\left(p: 10^{-4} \div 10^{-50}\right)$. Interestingly, this trend can be noticed also for TGM3 but only in melanoma and SCC samples $\left(p: 10^{-2} \div 10^{-50}\right)$, while the differentiationrelated pattern of TGM3 expression is completely lost in BCC samples (GO groups: "aromatic/nitrogen/lipid compound biosynthesis", "water-soluble vitamin metabolism", etc.; $p>10^{-3}$ ). These data indicate that, unlike other skin cancers, in BCC, despite the strong de-regulation of TG3 expression, other proteins related to keratinization are not altered.

\section{Discussion}

Skin differentiation is a specialized form of cell death, but unlike apoptosis, p53, Bcl2 [28-38] and other classic proapoptotic effectors are not involved, whereas TG enzymes (TG1, TG3, and TG5) play a crucial role [2, 4-6, 39]. TG3 is $\mathrm{a} \mathrm{Ca}^{2+}$-dependent enzyme, important for protein crosslinking. It is mainly expressed in squamous epithelia such as the epidermis, as well as in hair follicles. Its crosslink- ing activity contributes to the formation of the cornified cell envelope and hair shaft [3]. Interestingly, the skin of $\mathrm{Tgm}^{-/-}$mice shows higher permeability and is more sensitized to photodamage induced by UVB-irradiation $[8,9]$. Moreover, loss of TG3 was described in head and neck and oesophageal squamous cell carcinoma $[18,19]$, indicating its possible involvement in epithelial cancers. However, the role of TG3 in skin cancer remains unresolved, thus we investigated its expression with regards to this pathology.

Firstly, we performed immunohistochemical staining using a tissue micro-array, containing different types of skin cancer as well as normal skin controls. We observed positive staining for TG3 in the upper spinous and granular layers of normal epidermis, while basal layer cells as well as melanocytes were negative. Indeed, melanoma samples maintained negative staining for TG3 as expected due to the non-squamous origin of this type of skin cancer. Interestingly, most squamous cell carcinoma samples were negative for TG3 as well. The latter confirms previously published data $[18,19]$. Of note, SCCs that stained positively for TG3 were well differentiated around keratin pearls. Conversely, we found a very strong signal for TG3 in basal cell carcinoma samples. Surprisingly, in these samples, TG3 was detected both in the cytoplasm and nucleus. Since BCC carcinogenesis shares several common features 


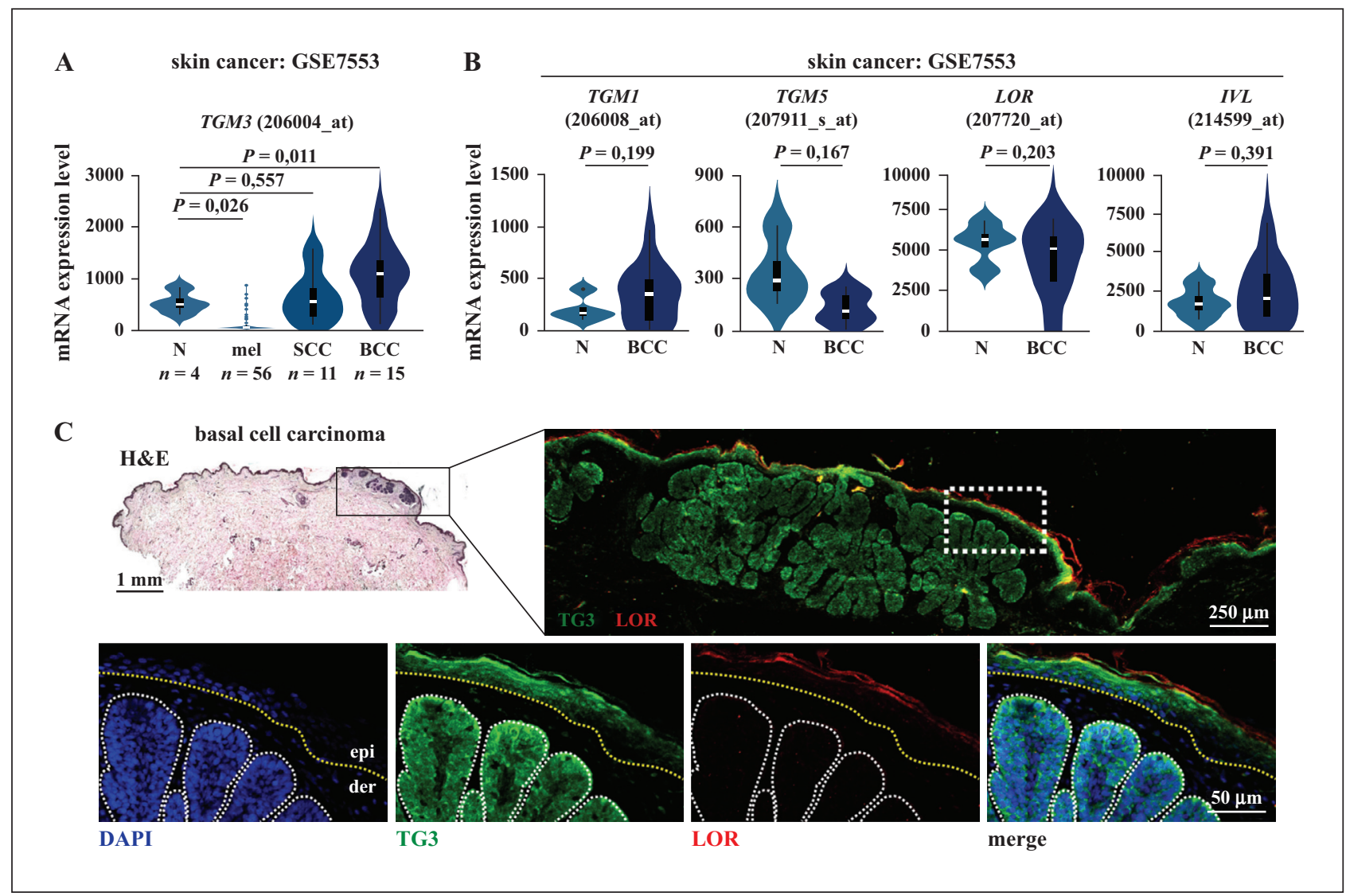

Figure 3. TGM3 is uniquely overexpressed in $\mathrm{BCC}$ relative to other TGs and its substrates. A) Violin plot showing relative mRNA expression of TGM3 in normal and skin cancer samples from GSE7553. B) Violin plot showing relative mRNA expression levels of TGM1, TGM5, LOR, and IVL in normal and BCC samples from GSE7553. C) Immunofluorescence analysis of TG3 and LOR expression in a case of BCC (the yellow dashed line separates the epidermis and dermis and white dashed lines indicate the tumour regions).

with hair development, additional analysis of TG3 expression and enzymatic activity should be performed during the initial steps of hair follicle development. Moreover, abnormal nuclear localisation of TG3 in tumour cells indicates a possible existence of additional activities of this enzyme which are uncommon in differentiated epidermal cells.

Further investigation of TGM3 expression at the mRNA level in skin cancer revealed a dramatic de-regulation of its differentiation-related pattern of expression only in BCC samples, and not in SCC or melanoma. Interestingly, the expression of other related genes, such as $T G M 1, T G M 5, L O R$, or $I V L$, strongly correlated with differentiation in all samples, hence de-regulation of TGM3 expression cannot be explained by aberrations in the differentiation programme, but implies the existence of additional transcriptional activities which trigger TGM3 expression exclusively during BCC tumourigenesis. More accurate analysis reveals that the cells of the basal layer of normal skin, where BCC originates from, are negative for TG3, in contrast to tumour cells. This observation indicates that during initiation of BCC, several pathways lead to transcriptional activation of TGM3 expression. BCCs are characterised by the altered Hedgehog pathway and, as a consequence, abnormal activity of GLI transcription factors [40]. Of interest, several binding sites for GLI2 were identified within the promoter region of TGM3 [41, 42]. Hence, a possible scenario of abnormal TG3 expression in $\mathrm{BCC}$ could be related to transcriptional activation of TGM3 by GLI2. However, the exact mechanisms for TG3 regulation remain completely elusive.

Of note, the pattern of TG3 expression is similar to that of Ep-CAM, specifically found in this type of skin cancer. In fact, routinely, BCC diagnosis is also confirmed by positive Ep-CAM staining [43]. Ep-CAM is absent in normal skin, and negative staining for Ep-CAM in tissue could be due to inappropriate fixation or processing of the samples, which may lead to uncertain conclusions regarding the diagnosis. As TG3 is expressed in both BCC cells and the granular layer of normal epidermis, TG3 staining of adjacent skin may serve as an internal positive control for antibody and sample preparation, rendering TG3 a potentially more accurate diagnostic marker for $\mathrm{BCC}$ relative to Ep-CAM.

Altogether, our findings demonstrate an abnormal overexpression of TG3 in basal cell carcinoma. Further research is necessary to support these observations with a greater number of samples, to reveal molecular mechanisms responsible 
A
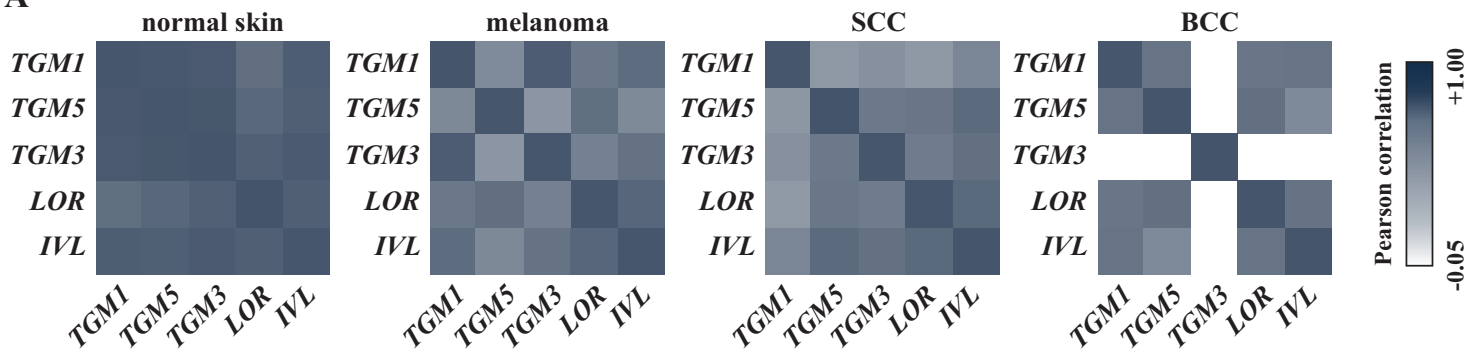

B
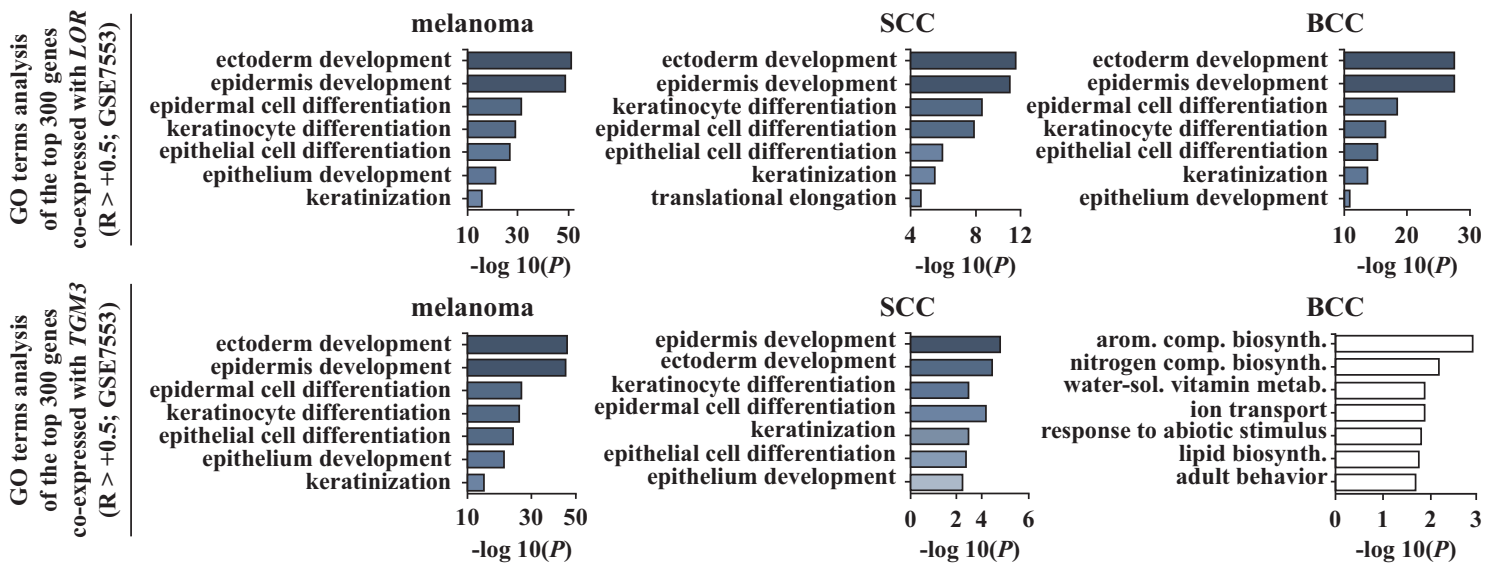

Figure 4. Differentiation-correlated profile of TGM3 expression is lost in BCC, but not in SCC. A) Heatmaps showing the correlation of expression between TGM1, TGM3, TGM5, LOR, and IVL in normal skin and different types of skin cancer based on the gene array, GSE7553. B) Gene ontology analysis showing the top 300 genes co-expressed with either LOR or TGM3 in melanoma, BCC, and SCC based on the gene array, GSE7553. Pearson's correlation R > +0.50.

for this phenomenon. The data presented indicate TG3 as a new potential specific marker for the diagnosis of cutaneous basal cell carcinoma.

Disclosure. Acknowledgements: We thank Caterina Marcelli, Dr. Manuel Scimeca, and Dr. Andrea Saggini (University Hospital "Policlinico Tor Vergata", Rome, Italy) for technical assistance and discussion. This work was mainly supported by IDI-IRCCS, RC to EC, and partially supported by a AIRC grant to EC (IG22206). The authors declare that they have no competing interests.

\section{Supplementary data}

Supplementary data (Table S1, Table S2, Table S3, Table S4) associated with this article can be found, in the online version, at doi:10.1684/ejd.2019.3636.

\section{References}

1. Lorand L, Graham RM. Transglutaminases: crosslinking enzymes with pleiotropic functions. Nat Rev Mol Cell Biol 2003; 4: 140-56.
2. Candi E, Melino G, Lahm A, et al. Transglutaminase 1 mutations in lamellar ichthyosis: loss of activity due to failure of activation by proteolytic processing. I Biol Chem 1998;273: 13693-702.

3. Candi E, Tarcsa E, Idler WW, Kartasova T, Marekov LN, Steinert PM. Transglutaminase cross-linking properties of the small proline-rich 1 family of cornified cell envelope proteins: integration with loricrin. J Biol Chem 1999; 274: 7226-37.

4. Candi E, Oddi S, Terrinoni A, et al. Transglutaminase 5 cross-links loricrin, involucrin, and small proline-rich proteins in vitro. J Biol Chem 2001; 276: 35014-23.

5. Kim IG, Lee SC, Lee JH, Yang JM, Chung SI, Steinert PM. Structure and organization of the human transglutaminase 3 gene: evolutionary relationship to the transglutaminase family. J Invest Dermatol 1994; 103: 137-42.

6. Lee JH, Jang SI, Yang JM, Markova NG, Steinert PM. The proximal promoter of the human transglutaminase 3 gene: stratified squamous epithelial-specific expression in cultured cells is mediated by binding of $\mathrm{Spl}$ and ets transcription factors to a proximal promoter element. J Biol Chem 1996; 271:4561-8.

7. Zocchi L, Terrinoni A, Candi $E$, et al. Identification of transglutaminase 3 splicing isoforms. J Invest Dermatol 2007; 127: 1791-4.

8. Bognar $\mathrm{P}, \mathrm{Nemeth} \mathrm{I}$, Mayer B, et al. Reduced inflammatory threshold indicates skin barrier defect in transglutaminase 3 knockout mice. J Invest Dermatol 2014; 134: 105-1 1

9. Frezza V, Terrinoni A, Pitolli C, Mauriello A, Melino G, Candi E. Transglutaminase 3 protects against photodamage. J Invest Dermatol 2017; 137: 1590-4.

10. Ü Basmanav FB, Cau L, Tafazzoli A, et al. Mutations in three genes encoding proteins involved in hair shaft formation cause uncombable hair syndrome. Am J Hum Genet 2016; 99: 1292-304. 
11. To G. Tumor-specific genetic expression profile of metastatic oral squamous cell carcinoma. Head Neck 2016;29: 1-18.

12. Choi $P$, Jordan $C D$, Mendez $E$, et al. Examination of oral cancer biomarkers by tissue microarray analysis. Otolaryngol Head Neck Surg 2008; 134: 539-46.

13. Lallemant $B$, Evrard $A$, Combescure $C$, et al. Clinical relevance of nine transcriptional molecular markers for the diagnosis of head and neck squamous cell carcinoma in tissue and saliva rinse. BMC Cancer 2009; $9: 370$

14. Wu $X$, Cao W, Wang $X$, et al. TGM3, a candidate tumor suppressor gene, contributes to human head and neck cancer. Mol Cancer $2013 ; 12: 151$.

15. Luo A, Kong J, Hu G, et al. Discovery of Ca2+-relevant and differentiation-associated genes downregulated in esophageal squamous cell carcinoma using cDNA microarray. Oncogene 2004; 23: 1291-9.

16. Bundela $S$, Sharma $A$, Bisen PS. Potential therapeutic targets for oral cancer: ADM, TP53, EGFR, LYN, CTLA4, SKIL, CTGF, CD70. PLoS ONE 2014; 9: e102610.

17. Nair J, Jain P, Chandola U, et al. Gene and miRNA expression changes in squamous cell carcinoma of larynx and hypopharynx. Genes Cancer 2015; 6: 328-40.

18. Negishi A, Masuda $M$, Ono $M$, et al. Quantitative proteomics using formalin-fixed paraffin-embedded tissues of oral squamous cell carcinoma. Cancer Sci 2009; 100: 1605-1 1.

19. Uemura N, Nakanishi $Y$, Kato $H$, et al. Transglutaminase 3 as a prognostic biomarker in esophageal cancer revealed by proteomics. Int J Cancer 2009; 124: 2106-15.

20. Li W, Zhang Z, Zhao W, Han N. Transglutaminase 3 protein modulates human esophageal cancer cell growth by targeting the NF$\kappa \mathrm{B}$ signaling pathway. Oncol Rep 2016; 36: 1723-30.

21. Peterson SC, Eberl $M$, Vagnozzi $A N$, et al. Basal cell carcinoma preferentially arises from stem cells within hair follicle and mechanosensory niches. Cell Stem Cell 2015; 16:400-12.

22. Khavari PA. Modelling cancer in human skin tissue. Nat Rev Cancer 2006; 6: 270-80.

23. Owens B. Melanoma. Nature 2014;515: S109.

24. Stacey $S N$, Sulem $P$, Gudbjartsson DF, et al. Germline sequence variants in TGM3 and RGS22 confer risk of basal cell carcinoma. Hum Mol Genet 2014; 23: 3045-53.

25. Riker Al, Enkemann SA, Fodstad $O$, et al. The gene expression profiles of primary and metastatic melanoma yields a transition point of tumor progression and metastasis. BMC Med Genomics 2008; 1: 13 .

26. Hitomi K, Presland RB, Nakayama T, Fleckman P, Dale BA, Maki $M$. Analysis of epidermal-type transglutaminase (transglutaminase 3 ) in human stratified epithelia and cultured keratinocytes using monoclonal antibodies. J Dermatol Sci 2003; 32: 95-103.
27. Dasgeb $B$, Mohammadi TM, Mehregan DR. Use of Ber-EP4 and epithelial specific antigen to differentiate clinical simulators of basal cell carcinoma. Biomark Cancer 2013; 5: BIC.S1 1856.

28. Aubrey BJ, Kelly GL, Janic A, Herold M, Strasser A. How does p53 induce apoptosis and how does this relate to p53-mediated tumour suppression? Cell Death Differ 2018; 25: 104-13.

29. Baugh $\mathrm{EH}, \mathrm{Ke} \mathrm{H}$, Levine $\mathrm{A}$, Bonneau RA, Chan CS. Why are there hotspot mutations in the TP53 gene in human cancers? Cell Death Differ 2018; 25: 154-60.

30. Charni M, Aloni-Grinstein R, Molchadsky A, Rotter V. P53 on the crossroad between regeneration and cancer. Cell Death Differ 2017; 24: 8-14

31. Engeland K. Cell cycle arrest through indirect transcriptional repression by p53: I have a DREAM. Cell Death Differ 2018; 25: $114-32$.

32. Sullivan KD, Galbraith MD, Andrysik Z, Espinosa JM. Mechanisms of transcriptional regulation by p53. Cell Death Differ 2018; 25: 133-43.

33. Galluzzi L, Vitale I, Aaronson SA, et al. Molecular mechanisms of cell death: recommendations of the Nomenclature Committee on Cell Death 2018. Cell Death Differ 2018; 25: 486-541.

34. Kim MP, Lozano G. Mutant p53 partners in crime. Cell Death Differ 2018; 25: 161-8.

35. Adams JM, Cory S. The BCL-2 arbiters of apoptosis and their growing role as cancer targets. Cell Death Differ 2018; 25: 27-36.

36. Kalkavan H, Green DR. MOMP, cell suicide as a BCL-2 family business. Cell Death Differ 2018; 25: 46-55.

37. Pihán $P$, Carreras-Sureda $A$, Hetz C. BCL-2 family: integrating stress responses at the ER to control cell demise. Cell Death Differ 2017; 24: 1478-87.

38. Strasser $A$, Vaux DL. Viewing $B C L 2$ and cell death control from an evolutionary perspective. Cell Death Differ 2018; 25: 13-20.

39. Ahvazi B, Boeshans KM, Idler W, Baxa U, Steinert PM. Roles of calcium ions in the activation and activity of the transglutaminase 3 enzyme. J Biol Chem 2003; 278: 23834-41.

40. Ikram MS, Neill GW, Regl G, et al. GLI2 is expressed in normal human epidermis and BCC and induces GLII expression by binding to its promoter. J Invest Dermatol 2004; 122: 1503-9.

41. Winklmayr $M$, Schmid $C$, Laner-Plamberger $S$, et al. Nonconsensus $\mathrm{GLI}$ binding sites in Hedgehog target gene regulation. BMC Mol Biol 2010; 11:2.

42. Laner-Plamberger $S$, Kaser $A$, Paulischta $M$, Hauser-Kronberger C, Eichberger T, Frischauf AM. Cooperation between GLI and JUN enhances transcription of JUN and selected GLI target genes. Oncogene 2009; 28: 1639-51.

43. Sunjaya AP, Sunjaya AF, Tan ST. The use of BEREP4 immunohistochemistry staining for detection of basal cell carcinoma. J Skin Cancer 2017; 2017: 2692604 . 